\title{
Building Laboratory-Based Arbovirus Sentinel Surveillance Capacity During an Ongoing Dengue Outbreak, Burkina Faso, 2017
}

Anselme Simeon Sanou, Emilio Dirlikov, Kongnimissom Apoline Sondo, Thérèse Samdapawindé Kagoné, Issaka Yameogo, Hyacinthe Euvrard Sow, Aimé Gilles Adjami, Siriky Martin Traore, Amadou Dicko, Bachirou Tinto, Eric Arnaud Diendere, Sonia Marie W. K. Ouedraogo-Konate, Tanga Kiemtore, David Tiga Kangoye, Lassana Sangare, Emilie T. H. Dama, James A. Fuller, Chelsea G. Major, Rafael Tosado-Acevedo, Tyler M. Sharp, Rebecca Greco Koné, and Brice Wilfried Bicaba

In West Africa, identification of nonmalarial acute febrile illness (AFI) etiologic pathogens is challenging, given limited epidemiologic surveillance and laboratory testing, including for AFI caused by arboviruses. Consequently, public health action to prevent, detect, and respond to outbreaks is constrained, as experienced during dengue outbreaks in several African countries. We describe the successful implementation of laboratory-based arbovirus sentinel surveillance during a dengue outbreak in Burkina Faso during fall 2017. We describe implementation, surveillance methods, and associated costs of enhanced surveillance during an outbreak response as an effort to build capacity to better understand the burden of disease caused by arboviruses in Burkina Faso. The system improved on existing routine surveillance through an improved case report form, systematic testing of specimens, and linking patient information with laboratory results through a data management system. Lessons learned will improve arbovirus surveillance in Burkina Faso and will

Anselme Simeon Sanou, MD, is Senior Surveillance Advisor; Emilie T. H. Dama, PhD, is Senior Laboratory Advisor; and Rebecca Greco Koné, MPH, is Country Director; all in the Division for Global Health Protection/Burkina Faso Country Office, US Centers for Disease Control and Prevention, Ouagadougou, Burkina Faso. Emilio Dirlikov, PhD, is Emergency Public Health Epidemiologist, Division for Global Health Protection/Emergency Recovery and Response Branch/Global Rapid Response Team; and James A. Fuller, $\mathrm{PhD}$, is an Epidemiologist, Division for Global Health Protection/Global Disease Detection Operations Center; both at the US Centers for Disease Control and Prevention, Atlanta, Georgia. Kongnimissom Apoline Sondo, MD, is Head of the Service, Service of Infectious Diseases; Eric Arnaud Diendere, MD, is Infectiologist, Service of Infectious Diseases; David Tiga Kangoye, MD, is an Immuno Epidemiologist, Head of the Department of Public Health; and Lassana Sangare, PhD, is Virologist, Head of the Department of laboratories; all at the Centre Hospitalier Universitaire Yalgado Ouedraogo, Ouagadougou, Burkina Faso. Thérèse Samdapawindé Kagoné, $\mathrm{PhD}$, is Head of the Viral Hemorrhagic Fever laboratory; Amadou Dicko, DVM, is a Researcher; and Bachirou Tinto, PharmD, is a Researcher; all at the Laboratoire National de Référence des Fièvres Hémorragiques Virales, Centre Muraz, BoboDioulasso, Burkina Faso. Issaka Yameogo, MD, is a Medical Epidemiologist; Hyacinthe Euvrard Sow, MD, is a Medical Epidemiologist; Siriky Martin Traore, PharmD, is a Medical Biologist; Sonia Marie W. K. Ouedraogo-Konate, MD, is a Medical Epidemiologist; Tanga Kiemtore, is Data Manager; and Brice Wilfried Bicaba, MD, is Director; all at the Direction de la Protection de la Santé de la Population, Ministère de la Sante, Ouagadougou, Burkina Faso. Aimé Gilles Adjami, PhD, is a Biologist and Executive Director, DAVYCAS International, Ouagadougou, Burkina Faso. Chelsea G. Major, MPH, is a Public Health Advisor; Rafael Tosado-Acevedo, PhD, is a Microbiologist; and Tyler M. Sharp, PhD, is an Epidemiologist; all at the National Center for Emerging and Zoonotic Infectious Diseases/Division of Vector-Borne Diseases/Dengue Branch, US Centers for Disease Control and Prevention, San Juan, Puerto Rico. 
contribute to enhancing global health security in the region. Elements critical to the success of this intervention include responding to a specific and urgent request by the government of Burkina Faso and building on existing systems and infrastructure already supported by CDC's global health security program.

Keywords: Epidemic management/response, Arbovirus, Dengue, Sentinel surveillance, Medical management/response

A RTHROPOD-BORNE VIRUSES, or arboviruses, are transmitted by arthropod vectors, such as mosquitoes, ticks, and fleas. Such viruses include yellow fever, chikungunya, West Nile, Zika, and dengue viruses. ${ }^{1}$ Globally, dengue is the most common arboviral disease. ${ }^{2}$ Dengue is caused by any of 4 closely related viruses (dengue virus [DENV] 1-4), which are transmitted by Aedes species mosquitoes. ${ }^{3}$ Most DENV infections are asymptomatic or result in acute febrile illness (AFI), with symptoms including headache, retro-orbital eye pain, myalgia, arthralgia, rash, and mild bleeding. ${ }^{4,5}$ However, infections may develop into lifethreatening conditions, including plasma leakage or severe hemorrhagic syndrome, ${ }^{6}$ as well as neurologic manifestations such as encephalitis and Guillain-Barré syndrome. ${ }^{7}$

Over one-third of the world lives in areas at risk of DENV transmission, including much of Africa. Although dengue epidemics have been reported in Africa since the late 19th century, the epidemiology and public health impact of dengue in Africa remains unclear due to limited surveillance and low awareness. ${ }^{8}$ DENV transmission is expected throughout much of Africa given the wide distribution of Aedes spp. mosquitoes, high population density, unplanned urbanization, and increased international travel. ${ }^{9,10}$ Local transmission is further evidenced by infection among travelers returning from Africa: Confirmed DENV infections were reported in travelers returning from 27 African countries where dengue was not considered endemic. $^{8,11-13}$ Detecting DENV infections in Africa is complicated by the high prevalence of malaria, which can present with similar AFI symptoms; an estimated $>70 \%$ of AFI cases in Africa are treated as presumptive malaria infections. ${ }^{14}$ Recent epidemiologic studies are beginning to fill gaps in knowledge about dengue in Africa. ${ }^{15-22}$

Burkina Faso is a sub-Saharan African country with an estimated population of 20 million people in $2018 .^{23}$ Burkina Faso has committed to the Global Health Security Agenda (GHSA), which was launched in 2014 to accelerate compliance with the 2005 International Health Regulations (IHR). ${ }^{24,25}$ As such, Burkina Faso receives technical and financial support from the US Centers for Disease Control and Prevention (CDC) and other global partners to strengthen capacity to prevent, detect, and respond to public health threats, including diseases that cause AFI. GHSA support has included establishing a Severe Acute Respiratory Illness (SARI) laboratory-based sentinel surveillance system, piloting an innovative system for transporting specimens using the National Society for Postal
Services (SONAPOST) from health facilities to the reference laboratory, and enhancing diagnostic capacity at the National Reference Laboratory for Viral Hemorrhagic Fevers (NRL-VHF).

While there is historic evidence of dengue endemicity in Burkina Faso, ${ }^{8,11,13,26-33}$ recent case surveillance and laboratory testing confirmed dengue circulation. ${ }^{24-27} \mathrm{Al}$ though dengue reporting was not compulsory, during a dengue outbreak in 2016, a total of 1,947 suspected dengue cases were identified, of which 1,327 (78\%) were positive by rapid diagnostic test (RDT), and additional laboratory testing of 35 patient specimens found 19 (54\%) confirmed DENV infections using RT-PCR: 11 were DENV-2, 6 were DENV-3, 2 were nontypeable, and 1 was a DENV-2/ DENV-3 co-infection. ${ }^{34}$ Beginning in August 2017, dengue cases were again identified in Burkina Faso, and on September 28, the Burkina Faso Ministry of Health $(\mathrm{MOH})$ declared a dengue epidemic. As part of the outbreak response, the Burkina Faso $\mathrm{MOH}, \mathrm{CDC}$, and DAVYCAS International collaborated with 2 healthcare centers in Ouagadougou to implement arbovirus sentinel surveillance.

In this report, we describe the surveillance system protocol, including prospective identification of arbovirus infections among outpatient and hospitalized AFI cases, laboratory testing, clinical chart abstraction, and data management. We discuss challenges and lessons learned, toward establishing evidence-based best practices to enhance global health security in the region and further afield.

\section{Emergency Response Dengue Sentinel \\ Surveillance}

\section{Objectives}

The overall aim of the sentinel surveillance system was to enhance knowledge to guide the Burkina Faso $\mathrm{MOH}$ for targeted public health action regarding dengue and other arboviruses circulating in Burkina Faso. More specifically, there were 4 primary objectives: (1) describe dengue cases, including the clinical course of hospitalized patients; (2) provide descriptive epidemiologic analysis to inform public health action; (3) provide laboratory-based diagnostic testing to define the frequency of DENV infection among patients presenting with AFI at the 2 sentinel sites; and (4) 
describe the frequency of severe dengue among hospitalized patients who presented during the ongoing 2017 dengue outbreak. More broadly, the sentinel surveillance system aimed to systematically collect case-based laboratory data on DENV and other potentially circulating pathogens of importance to global health security.

AFI may be caused by a variety of pathogens, some of which are highlighted by GHSA, including arboviruses such as dengue, yellow fever, West Nile, and Zika viruses. Appropriate national, regional, and even global responses require thorough case investigations linked to laboratory detection. Given recent outbreaks of Zika virus in the Western Pacific and Americas ${ }^{35}$ and yellow fever virus in Angola and the Democratic Republic of Congo ${ }^{35}$ as well as Brazil, ${ }^{36}$ during the 2017 outbreak in Burkina Faso casebased laboratory surveillance was crucial to confirm dengue and exclude other potentially circulating arboviruses.

\section{Sentinel Site Selection and Description}

The sentinel surveillance system was implemented in 2 sites in Ouagadougou, the capital of Burkina Faso and part of the Central Health Region. The first site was NongrMassom District Hospital, one of 4 district hospitals in the Central Health Region that comprises 16 units, including surgery, maternity, pediatrics, and medicine. It consists of 95 beds for hospitalization and 130 health workers. Nongr-Massom District Hospital was selected as a sentinel surveillance site due to its large population catchment (estimated at 365,133 persons), as well as the large burden of dengue cases among outpatients reported to the national syndromic surveillance system.

The second site was Yalgado Ouedraogo University Hospital Center (Yalgado CHU), a tertiary referral hospital located in Ouagadougou. ${ }^{37}$ It comprises 10 departments divided into 47 clinical and medical technical services providing care and diagnostic services, as well as conducting clinical training and research activities. Yalgado $\mathrm{CHU}$ has 713 beds and 1,400 staff, with approximately 24,000 to 25,000 consultations per year. The infectious disease department has 25 beds and attends to an average of 250 patients per year. Patients generally are admitted to the department following consultation at Yalgado $\mathrm{CHU}$ emergency department, direct consultation of a febrile patient to the infectious disease department, or indirect referrals from outside public and private health facilities. Most outpatient consultations are for non-malaria AFI, including dengue. The Yalgado $\mathrm{CHU}$ infectious disease department has been identified by Burkina Faso $\mathrm{MOH}$ as a reference center for severe dengue cases from the Central and surrounding health regions. As such, clinicians have been deeply involved with the development of national dengue case management guidelines, using experience garnered during the 2016 dengue epidemic and the results of research activities conducted to better understand clinical aspects of dengue in Burkina Faso.

\section{National Reference Laboratory for Viral Hemorrhagic Fevers}

Laboratory testing was conducted at the NRL-VHF based at an $\mathrm{MOH}$ public health research center, Centre Muraz in Bobo-Dioulasso. ${ }^{38}$ The laboratory was established in 2014 as part of the response to the Ebola outbreak in West Africa and has since been supported by CDC and other partners to enhance capacity to detect viral pathogens identified as priorities by the Burkina Faso MOH. In addition to Ebola virus, the laboratory can test for a range of arboviruses (eg, dengue, chikungunya, Zika, yellow fever), as well as Rift Valley fever, Crimea-Congo hemorrhagic fever, and Lassa fever viruses. In 2017, the NRL-VHF became the Regional Reference Laboratory for Viral Hemorrhagic Fevers for the Economic Community of West African States (ECOWAS). ${ }^{39}$ The acquisition of a Biological Safety Level (BSL)-3 mobile laboratory increases the NRL-VHF's capacity to safely operate during potential future emergency responses to viral hemorrhagic fevers. In addition to research activities, the NRL-VHF has supported Burkina Faso MOH in the detection and response to disease outbreaks, including during the 2016 and 2017 dengue outbreaks.

\section{Clinical Case Definitions and Case Reporting}

A case report form (see Appendix 1, supplemental material, at https://www.liebertpub.com/doi/suppl/10.1089/hs. 2018.0048) was developed using the 2017 Pan-American Health Organization/World Health Organization (PAHO/ WHO) clinical case definition for dengue without warning signs (DNWS), dengue with warning signs (DWWS), and severe dengue (SD) (Figure 1). ${ }^{40}$ Descriptive epidemiologic variables included age, sex, place of residence, and place of employment. Additional relevant variables included recent travel history, reporting recent contact with living or dead animals, and reporting recent mosquito bites.

Case identification differed slightly between the 2 sentinel sites. At Nongr-Massom District Hospital, any patient who was seen at any department could be reported if they had a fever (subjective or objective) and at least 2 of the symptoms present in the $2017 \mathrm{PAHO} / \mathrm{WHO}$ clinical case definition (Figure 1). ${ }^{40}$ Chief complaint of diarrhea or cough were used as exclusion criteria. At Yalgado CHU, outpatient and hospitalized cases in the infectious disease, emergency, nephrology, pediatric, gynecology, and obstetrical departments that met clinical case definitions were reported, and chart abstraction was conducted to collect data on all patients who were hospitalized in 2017 (Appendix 2, supplemental material, at https:/www.liebertpub.com/doi/ suppl/10.1089/hs.2018.0048). 
DENGUE WITHOUT WARNING SIGNS (DNWS)

Persons who lives or who has traveled to area with dengue transmission in the last 14 days and presents fever, usually of 27 days duration, and at least 2 of the following criteria:

1. Nausea/vomiting

2. Rash (exanthema)

3. Headache/retro-orbital pain

4. Muscle pain (myalgia)/joint pain (arthralgia)

5. Petechiae or positive tourniquet test

6. Leukopenia

Also, any child from or residing in a dengue transmission area with acute fever, usually $2-7$ days duration, with no apparent focus.
DENGUE WITH WARNING SIGNS (DWWS)

Any dengue case which at the time fever subsides presents with 1 or more of the following signs:

1. Intense abdominal pain or tenderness

2. Persistent vomiting

3. Fluid accumulation (ascites, plural, and/or pericardiac effusion)

4. Mucosal bleeding

5. Lethargy/restlessness

6. Postural hypotension (lipothymia)

7. Liver enlargement $>\mathbf{2} \mathrm{cm}$

8. Progressive increase in hematocrit

\section{SEVERE DENGUE (SD)}

Any dengue case with 1 or more of the following manifestations:

1. Shock or respiratory distress due to severe plasma leakage. Shock evidenced by weak or undetectable pulse, tachycardia, cold extremities, and capillary perfusion $>\mathbf{2}$ seconds, pulse pressure $<20 \mathrm{mmHg}$, indicating hypotension in the late phase

2. Severe bleeding based on evaluation by the attending physician (e.g., hematemesis, melena, ample metorrhagia, central nervous system [CNS] bleeding).

3. Severe organ compromise, such as liver impairment (AST or ALT $>1000 \mathrm{IU}$ ), CNS (impaired mental state), heart (myocarditis), or other organs

REQUIRES STRICT OBSERVATION AND MEDICAL INTERVENTION

Laboratory Testing and Laboratory-Based Case Definitions

At both sites, patient serum specimens were collected for laboratory testing. Site laboratory staff performed dengue RDT using the SD BIOLINE Dengue Duo, ${ }^{41}$ as well as malaria RDT, as per routine testing for AFI. Results for NS1 antigen and anti-DENV immunogloblulin M (IgM) and $\operatorname{IgG}$ were recorded on the case report form. Using refrigerators provided, the remaining patient specimens were stored at 2 to $8^{\circ} \mathrm{C}$ and collected twice a week by SONAPOST for transport to NRL-VHF. From October 31, 2017, to April 4, 2018, SONAPOST transported 247 specimens from the sentinel sites to NRL-VHF.

At NRL-VHF, patient specimens were tested using the Trioplex real-time reverse transcriptase-polymerase chain reaction (rRT-PCR) assay to detect evidence of current infection with dengue, chikungunya, and Zika viruses. ${ }^{42}$ Specimens were also tested by IgM enzyme-linked immunosorbent assay (ELISA) to detect anti-DENV, antiCHIKV, and anti-ZIKV IgM antibodies. CDC recommended that specimens be tested by rRT-PCR and ELISA using the following diagnostic algorithm based on date of specimen collection post-illness onset:

- 0-4 days: rRT-PCR only

- 4-7 days: both rRT-PCR and IgM ELISA

- $>7$ days: IgM ELISA only
Patients with nucleic acids detected by rRT-PCR for any of the arboviruses tested were considered to have confirmed infections. People with negative results for all arboviruses by rRT-PCR were considered to have presumptive recent infection with a single arbovirus when results were positive for that virus by IgM ELISA, and presumptive flavivirus infection if they tested positive for both DENV and ZIKV by IgM ELISA, given the possibility of cross-reactivity. After a pilot period, a specimen reception protocol was established to ensure all testing results could be linked to patients, using minimal required variables completed on the case report form. Verification of case report form completeness checks were conducted at the sites' laboratory and data entry units, and specimens missing required variables were rejected for testing by NRL-VHF.

\section{Implementation of Activities}

Implementation of activities took place primarily in November 2017, following a formal request for assistance from the Burkina Faso $\mathrm{MOH}$ to CDC. Coordination and technical meetings were organized among the Burkina Faso $\mathrm{MOH}, \mathrm{CDC}$, and DAVYCAS International. A surveillance protocol was developed by all stakeholders and was reviewed by the Burkina Faso $\mathrm{MOH}$ and $\mathrm{CDC}$ institutional review boards (IRB), which determined the protocol involved nonresearch emergency public health activities. Following a meeting with key high-level stakeholders from 
the Burkina $\mathrm{MOH}, \mathrm{CDC}$, DAVYCAS International, and leaders from both sites, half-day site-specific training for clinicians, laboratory technicians, and hospital administrators was conducted in late November. Additional standard operation procedures, such as specimen preparation procedures, were developed to guide in-field activities.

CDC technical assistance to NRL-VHF was provided through the CDC Burkina Faso Country Office as well as visits from laboratory experts in November and December 2017 and January 2018. Assistance focused on specimen management, testing quality control, and data management. Laboratory reagents and equipment were also donated by CDC to ensure timely specimen testing.

\section{Data Management and Analysis}

EpiInfo (version 7) databases were developed based on the case report form. In order to minimize the data management challenges, site-specific databases were developed for each of the sentinel sites and the NRL-VHF for entry and storage of relevant data; data from the sentinel sites and

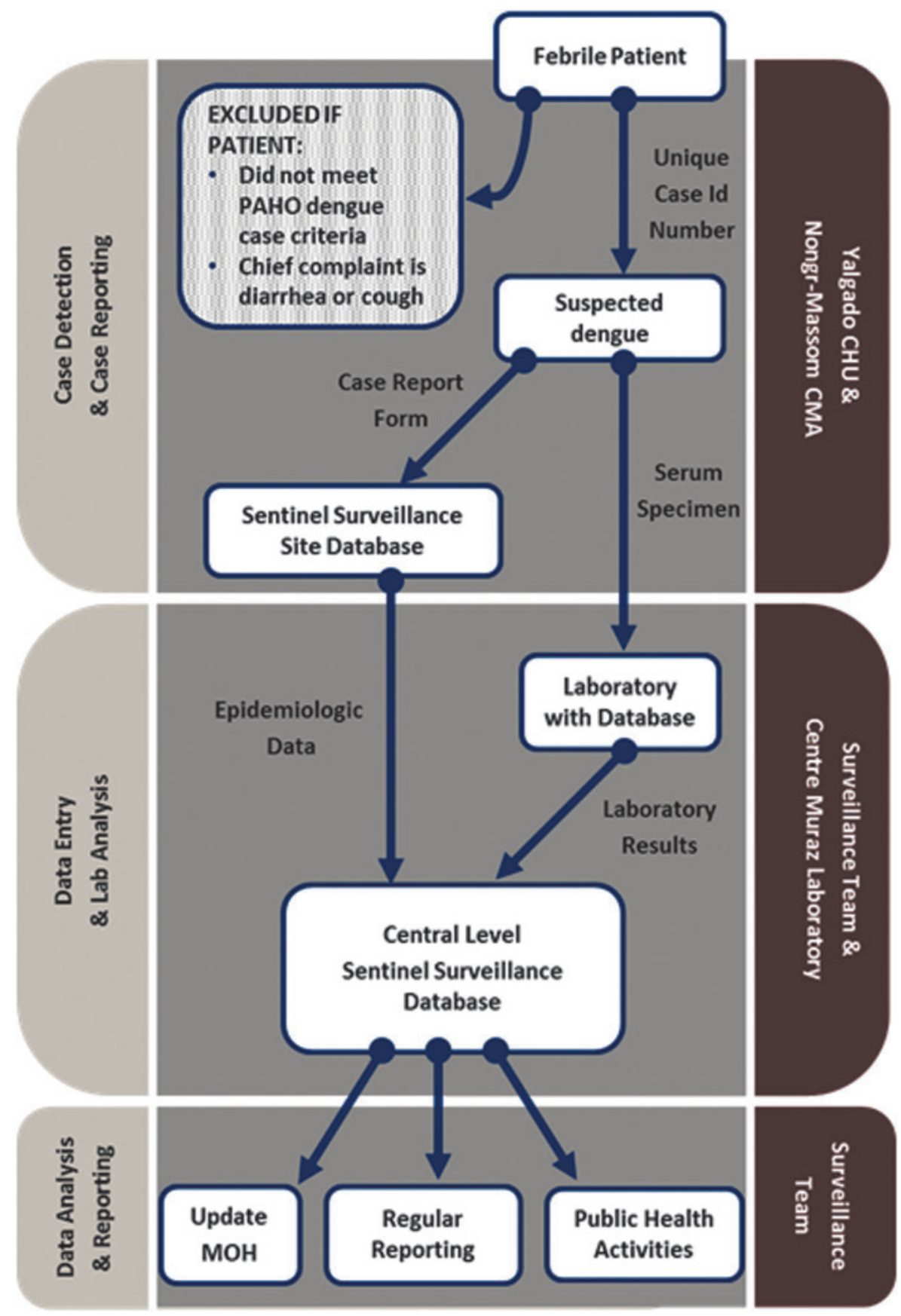

Figure 2. Case-Reporting Flow for Arbovirus Sentinel Surveillance in Yalgado University Hospital Center and Nongr-Massom District Hospital from October 31, 2017 to April 4, 2018 
NRL-VHF were sent weekly to the MOH surveillance team. Unique case identification numbers were assigned via barcode stickers, which were developed by DAVYCAS International. Stickers with matching case numbers were placed on case report forms and specimen tubes; case numbers were entered into databases manually until barcode scanners could be implemented at sites. After case report forms were completed manually by clinicians, and copies of the case report forms were made prior to specimen shipment to NRL-VHF. Data on case demographics, clinical characteristics, dengue RDT results, and specimen collection were entered into the sentinel site databases. Case report form sections on specimen receipt and diagnostic testing were completed by NRL-VHF staff and entered into the laboratory database, along with case number and other critical case identification and definition variables. The Burkina Faso MOH surveillance team used the unique case numbers to merge datasets sent from the sentinel sites and NRL-VHF for data cleaning and analysis (Figure 2).

\section{Leveraging Resources and Investment}

Establishing a laboratory-based sentinel surveillance system was intended to be an effective tool to provide high-quality and timely data for the Burkina Faso $\mathrm{MOH}$ to guide public health action. To do this effectively during an outbreak requires investment of new resources but, more importantly, depends on the ability to leverage existing systems and investments. Since committing to the GHSA in 2015, the Burkina Faso $\mathrm{MOH}$ has put into place or reinforced routine surveillance for epidemic prone diseases, which the ministry was able to draw on as a part of the dengue outbreak response. Specific systems and developed resources include an innovative, effective, and rapid specimen transport system implemented by the Burkina Faso $\mathrm{MOH}$ and SONAPOST, enhanced diagnostic capacity including reagents and equipment, extensive experience with the internationally recognized meningitis surveillance system and associated data management system, and human resources trained via the Field Epidemiology Training Program $(\text { FETP })^{43}$ with experience establishing a laboratory-based SARI sentinel surveillance system.

In addition to leveraging these existing resources, partners provided additional financial and technical support to the Burkina $\mathrm{MOH}$ to implement arbovirus sentinel surveillance during the dengue outbreak. Total direct financial support amounted to $\$ 16,631.49$ (8,842,850 CFA francs) over 4 months to provide training, specimen transport, and monitoring of sites. Total training costs were $\$ 6,068.41$ (36\% of total costs), with $\$ 4,723.25$ for on-site trainings and $\$ 1,345.16$ for data management training sessions.

Specimen transport from Ouagadougou to the NRLVHF in Bobo-Dioulasso cost $\$ 3,463.46$ (21\% of total costs). Support to ensure daily monitoring of sites and assure quality of data amounted to $\$ 3,715.13$ (19\% of total costs). The remaining costs were for material support, including 2 refrigerators for specimen storage $(\$ 2,165.72$ for both) and 2 photocopiers ( $\$ 1,412.42$ for both) for sentinel sites, and other associated materials. In addition to this direct financial contribution, partners also donated diagnostic reagents and dengue RDTs and provided expert technical consultations, both onsite in Burkina Faso and remotely via teleconference or email.

\section{Discussion}

In November 2017, sentinel arbovirus surveillance was rapidly designed and implemented through a coordinated effort among the Burkina Faso MOH, CDC, and DAVYCAS International. The capacity built through this effort will contribute to understanding the characteristics and burden of arboviral disease, so that targeted public health interventions to prevent further disease can be defined and implemented. Moreover, by leveraging new and existing resources to establish this sentinel surveillance, Burkina Faso has efficiently acquired more knowledge and capacity to detect emerging pathogens that could be a public health threat to the region. The sentinel surveillance improved data collection through a case report form, systematic testing of specimens, and linking patient information with laboratory results through the data management system. Collected epidemiologic, clinical, and laboratory data are helping to better understand the arbovirus infection and AFI that caused the 2017 dengue outbreak and can be used to prevent future outbreaks or quickly detect and identify emerging outbreaks.

Mobilization of existing partnerships and systems strengthened or established under GHSA were also crucial in rapidly implementing the surveillance system. As mentioned, effective sentinel surveillance design drew on protocols and best practices for SARI sentinel surveillance and the SONAPOST specimen transport system. This included use of stickers to easily link case report forms and patient specimens, as well as previously developed specimen transport protocols. Furthermore, during nonepidemic times, partnerships have built local emergency public health capacity to strengthen outbreak response. Through the CDC-sponsored FETP training, ${ }^{43} 1$ central level staff member had completed his advanced FETP program in July 2017, 2 staff members from Nongr-Massom District Hospital participated in the Frontline FETP in February 2017, and 1 staff member at Yalgado CHU completed Frontline FETP training in September 2016. Furthermore, a central level MOH staff received training through CDC's Atlanta-based public health emergency management (PHEM) fellowship. ${ }^{44}$

Moreover, success was greatly aided by partners' support to prevent and detect dengue and other arboviruses provided during nonepidemic periods. Following the 2016 dengue epidemic, the Burkina Faso $\mathrm{MOH}$ worked closely with partners, including CDC and $\mathrm{WHO}$, to better interpret public health data and improve outbreak response. This included high-level meetings held in February 2017 to distill key lessons learned. Finally, CDC has supported the 
NRL-VHF through technical training and provision of equipment and reagents to enhance viral diagnostics and improve biosafety environment and practices, including arbovirus testing using the Trioplex and DENV multiplex RT-PCR assays.

This outbreak response highlights the interrelation of activities focused on disease prevention, detection, and response. Through careful planning, dengue response activities in Burkina Faso drew on existing resources to improve the public health system's ability to detect dengue and other arboviruses. In March 2018, the experience of arbovirus sentinel surveillance was presented at a partner meeting led by WHO that brought together representatives from countries across the West Africa subregion and key partners such as the West Africa Health Organization. Lessons learned will be used to expand routine sentinel surveillance in 2018 in Burkina Faso and improve arbovirus case reporting nationally. While emergency public health response requires timely action, proper planning and review of experiences can help support prevention and detection activities during nonepidemic times.

Despite these achievements, several challenges and limitations bear mention. First, the surveillance system was unable to incorporate entomologic surveillance, which could have added an important component to guide vector control activities. Such activities are being considered as the sentinel surveillance system expands to new sites. Second, data management presented an ongoing challenge, especially given limited time for end-user testing in the field. Modifications to the data management system will improve data quality by refining internal quality control measures and the structure of the databases. Use of digital scanners to read stickers will improve entry of unique case numbers. Finally, the surveillance system was implemented after the peak of the outbreak, which will limit data analysis. Delays were attributed to required procedural steps and ethical reviews, as well as material preparations for implementation.

Overall, the Burkina Faso $\mathrm{MOH}$ was able to mobilize existing partnerships and resources toward the rapid implementation of arbovirus sentinel surveillance among AFI cases during a dengue outbreak. Information gathered will render useful information on the outbreak, including data on additional arboviruses potentially circulating and analysis of clinical features and case management of dengue in Burkina Faso focused on severe, hospitalized cases. The rich experience garnered will be used to implement sustainable routine AFI surveillance, given expected arbovirus endemicity, and has the opportunity to enhance Burkina Faso's ability to detect other viral pathogens of epidemic potential.

\section{REFERENCES}

1. National Center for Emerging and Zoonotic Diseases; Centers for Disease Control and Prevention. About the Division of Vector-Borne Diseases. CDC website. Updated
June 18, 2018. https://www.cdc.gov/ncezid/dvbd/about. html. Accessed August 29, 2018.

2. National Center for Emerging and Zoonotic Diseases; Centers for Disease Control and Prevention. About the Division of Vector-Borne Diseases. CDC website. Updated August 16, 2018. https://www.cdc.gov/ncezid/dvbd/index. html. Accessed August 29, 2018.

3. Halstead SB. Dengue. Lancet 2007;370(9599):1644-1652. Review.

4. Centers for Disease Control and Prevention. Dengue. CDC website. Updated March 28, 2016. https://wwwnc.cdc.gov/ travel/diseases/dengue. Accessed August 29, 2018.

5. Centers for Disease Control and Prevention. Dengue. CDC website. Updated January 19, 2016. https://www.cdc.gov/ dengue/. Accessed August 29, 2018.

6. Gubler DJ. Dengue and dengue hemorrhagic fever. Clin Microbiol Rev 1998;11(3):480-496.

7. Solomon T, Dung NM, Vaughn DW, et al. Neurological manifestations of dengue infection. Lancet 2000;355(9209): 1053-1059.

8. Amarasinghe A, Kuritsk JN, Letson GW, Margolis HS. Dengue virus infection in Africa. Emerg Infect Dis 2011; 17(8):1349-1354.

9. Stanaway JD, Shepard DS, Undurraga EA, et al. The global burden of dengue: an analysis from the Global Burden of Disease Study 2013. Lancet Infect Dis 2016;16(6):712723.

10. Kraemer MU, Sinka ME, Duda KA, et al. The global distribution of the arbovirus vectors Aedes aegypti and Ae. albopictus. Elife 2015;4:e08347.

11. Eldin C, Gautret P, Nougairede A, et al. Identification of dengue type 2 virus in febrile travellers returning from Burkina Faso to France, related to an ongoing outbreak, October to November 2016. Euro Surveill 2016;21(50).

12. Franco E, Bagnato B, Marino MG, Meleleo C, Serino L, Zaratti L. Hepatitis B: epidemiology and prevention in developing countries. World J Hepatol 2012;4(3):74-80.

13. Eisenhut M, Schwarz TF, Hegenscheid B. Seroprevalence of dengue, chikungunya and Sindbis virus infections in German aid workers. Infection 1999;27(2):82-85.

14. Stoler J, Al Dashti R, Anto F, Fobil JN, Awandare GA. Deconstructing "malaria": West Africa as the next front for dengue fever surveillance and control. Acta Trop 2014;134: 58-65.

15. Sharp C, Venta A, Marais L, Skinner D, Lenka M, Serekoane J. First evaluation of a population-based screen to detect emotional-behavior disorders in orphaned children in Sub-Saharan Africa. AIDS Behav 2014;18(6):1174-1185.

16. Blaylock JM, Maranich A, Bauer K, et al. The seroprevalence and seroincidence of dengue virus infection in western Kenya. Travel Med Infect Dis 2011;9(5):246-248.

17. Ellis EM, Neatherlin JC, Delorey M, et al. A household serosurvey to estimate the magnitude of a dengue outbreak in Mombasa, Kenya, 2013. PLoS Negl Trop Dis 2015;9(4): e0003733.

18. Konongoi L, Ofula V, Nyunja A, et al. Detection of dengue virus serotypes 1, 2 and 3 in selected regions of Kenya: 20112014. Virol J 2016;13(1):182.

19. Lutomiah J, Barrera R, Makio A, et al. Dengue outbreak in Mombasa City, Kenya, 2013-2014: entomologic investigations. PLoS Negl Trop Dis 2016;10(10):e0004981. 
20. Ochieng C, Ahenda P, Vittor AY, et al. Seroprevalence of infections with dengue, Rift Valley fever and chikungunya viruses in Kenya, 2007. PLoS One 2015;10(7):e0132645.

21. Vu DM, Banda T, Teng CY, et al. Dengue and West Nile virus transmission in children and adults in coastal Kenya. Am J Trop Med Hyg 2017;96(1):141-143.

22. Vu DM, Mutai N, Heath CJ, et al. Unrecognized dengue virus infections in children, western Kenya, 2014-2015. Emerg Infect Dis 2017;23(11):1915-1917.

23. Institut National de la Statistique et de la Démographie. Chiffres clés de l'Institut National de la Statistique et de la Démographie du Burkina Faso. 2018. http://www.insd.bf/n/. Accessed August 29, 2018.

24. Angulo FJ, Cassell CH, Tappero JW, Bunnell RE. Progress and opportunities for strengthening global health security. Emerg Infect Dis 2017;23(Suppl):S1-S4.

25. Fitzmaurice AG, Mahar M, Moriarty LF, et al. Contributions of the US Centers for Disease Control and Prevention in implementing the Global Health Security Agenda in 17 partner countries. Emerg Infect Dis 2017;23(Suppl):S15-S24.

26. Gonzalez JP, Du Saussay C, Gautun J, McCormick JB, Mouchet J. La dengue au Burkina Faso (ex-Haute-Volta): épidémies saisonnières en milieu urbain à Ouagadougou. Bull Soc Pathol Exot Filiales 1985;78(1):7-14.

27. Hervy J-P, Legros F, Roche JC, Monteny N, Diaco B. Circulation du virus dengue 2 dans plusieurs milieux boisés des savanes soudaniennes de la région de Bobo-Dioulasso (Burkina Faso): considérations entomologiques et épidémiologiques. Cahiers ORSTOM Série Entomologie Médicale et Parasitologie 1984;22(2):135-143.

28. Robert V, Lhuillier M, Meunier D, et al. [Yellow fever virus, dengue 2 and other arboviruses isolated from mosquitos, in Burkina Faso, from 1983 to 1986. Entomological and epidemiological considerations]. Bull Soc Pathol Exot 1993;86(2):90-100.

29. Collenberg E, Ouedraogo T, Ganamé J, et al. Seroprevalence of six different viruses among pregnant women and blood donors in rural and urban Burkina Faso: a comparative analysis. J Med Virol 2006;78(5):683-692.

30. Fournet F, Rican S, Vaillant Z, et al. The influence of urbanization modes on the spatial circulation of flaviviruses within Ouagadougou (Burkina Faso). Int $J$ Environ Res Public Health 2016;13(12):E1226.

31. Ridde V, Agier I, Bonnet E, et al. Presence of three dengue serotypes in Ouagadougou (Burkina Faso): research and public health implications. Infect Dis Poverty 2016;5:23.

32. Ridde V, Carabali M, Ly A, et al. The need for more research and public health interventions on dengue fever in Burkina Faso. PLoS Negl Trop Dis 2014;8(6):e2859.
33. Tarnagda Z, Congo M, Sagna T, et al. Outbreak of dengue fever in Ouagadougou, Burkina Faso, 2013. Int J Microbiol Immunol Res 2014;2:101-108.

34. Tarnagda Z, Cissé A, Bicaba BW, et al. Dengue fever in Burkina Faso, 2016. Emerg Infect Dis 2018;24(1):170-172.

35. Hills SL, Fischer M, Petersen LR. Epidemiology of Zika virus infection. J Infect Dis 2017;216(Suppl 10):S868-S874.

36. Moreira-Soto A, Torres MC, Lima de Mendonça MC, et al. Evidence for multiple sylvatic transmission cycles during the 2016-2017 yellow fever virus outbreak, Brazil. Clin Microbiol Infect 2018;24(9):1019.e1-1019.e4.

37. Centre Hospitalier Universitaire Yalgado Ouédraogo. http:// chuyobf.org/?page_id=5. Accessed August 29, 2018.

38. Centre MURAZ. http://www.centre-muraz.bf/index.php/en/. Accessed August 29, 2018.

39. Economic Community of West African States (ECOWAS). Member states. http://www.ecowas.int/member-states/. Accessed August 29, 2018.

40. Pan American Health Organization. Tool for the diagnosis and care of patients with suspected arboviral diseases; 2016. http://www.paho.org/hq/index.php?option=com_docman $\&$ task=doc_details\&gid=37524\&Itemid=270\&lang=en. Accessed August 29, 2018.

41. Wang SM, Sekaran SD. Early diagnosis of dengue infection using a commercial Dengue Duo rapid test kit for the detection of NS1, IGM, and IGG. Am J Trop Med Hyg 2010; 83(3):690-695.

42. Munoz-Jordan JL. Diagnosis of Zika virus infections: challenges and opportunities. J Infect Dis 2017;216(Suppl 10): S951-S956.

43. Jones DS, Dicker RC, Fontaine RE, et al. Building global epidemiology and response capacity with field epidemiology training programs. Emerg Infect Dis 2017;23(Suppl):S158S165.

44. Brencic DJ, Pinto M, Gill A, Kinzer MH, Hernandez L, Pasi OG. CDC support for global public health emergency management. Emerg Infect Dis 2017;23(Suppl):S183-S189.

Address correspondence to: Emilio Dirlikov, PhD Emergency Public Health Epidemiologist Emergency Response and Recovery Branch Division of Global Health Protection Center for Global Health Centers for Disease Control and Prevention Atlanta, GA

Email: KLT9@cdc.gov 